\title{
Improving the Accessibility of NFC/RFID-based Mobile Interaction through Learnability and Guidance
}

\author{
Gregor Broll ${ }^{1}$, Susanne Keck ${ }^{2}$, Paul Holleis ${ }^{1}$, Andreas Butz $^{2}$ \\ ${ }^{1}$ DOCOMO Euro-Labs, München, Germany \\ \{broll, holleis\}@docomolab-euro.com \\ ${ }^{2}$ Media Informatics Group, Ludwig-Maximilians-Universität (LMU), München, Germany \\ keck@cip.ifi.Imu.de, andreas.butz@ifi.Imu.de
}

\begin{abstract}
NFC and RFID technologies have found their way into current mobile phones and research has presented a variety of applications using NFC/RFID tags for interaction between physical objects and mobile devices. Since this type of interaction is widely novel for most users, there is a considerable initial inhibition threshold for them. In order to get novice users started with this physical interaction and its applications, we have designed different ways to increase the learnability and guidance of such applications. Their effectiveness was evaluated in a qualitative and quantitative user study with 40 participants, who interacted with NFC-equipped posters in different ways. We report on the types of usage errors observed and show that future designs of NFC/RFID-based mobile applications should consider using a dedicated start-tag for interaction.
\end{abstract}

\section{Categories and Subject Descriptors}

H.5.2 [Information Interfaces and Presentation]: User Interfaces - evaluation/methodology, input devices and strategies, interaction styles

\section{General Terms}

Design, Human Factors

\section{Keywords}

NFC, RFID, Physical Mobile Interaction, Multi-Tag Interaction, learnability, guidance, accessibility, usability, evaluation

\section{INTRODUCTION}

During the last years, technologies for short-range, contactless identification, data-exchange and interaction like Radio Frequency Identification (RFID) and Near Field Communication (NFC) have gained a lot of attention in industry and mobile computing research [17]. Both technologies use active reading devices to gather data stored on passive, wireless tags that can be attached to arbitrary objects. RFID is best known for the tagging of consumer products and its use in contactless smartcards for identification, ticketing or mobile payment. NFC is based on the same technology as RFID, but is better tailored to mobile devices. With the increasing availability of mobile phones that support

Copyright is held by the author/owner(s).

MobileHCI’09, September 15 - 18, 2009, Bonn, Germany.

ACM 978-1-60558-281-8.
NFC (e.g. Nokia 6131 NFC), it is likely to become the preferred technology for contactless mobile interaction.

A distinct property of NFC/RFID-based interaction is the short operating distance between devices and (passive) tags - about 3 to $5 \mathrm{~cm}$. Interaction is accomplished by touching tags with reading devices or holding them closely together. This physical interaction conveys the usage of the underlying technologies in an easily comprehensible way as it adopts the metaphor of interacting with something by simply touching it. The simplicity and directness of this physical interaction make NFC and RFID popular technologies for many mobile applications. One of many examples is SmartTouch, a European project that has investigated the usage of NFC in a variety of different use cases, such as ticketing, access control, home care or entertainment [14]. In mobile computing research, NFC and RFID are used for mobile interaction with tagged physical objects and associated digital resources, supporting the vision of Ubiquitous Computing [18].

NFC and RFID are highly suitable to facilitate mobile interaction due to the simplicity, directness and intuitiveness of the touchmetaphor. However, there is also a considerable inhibition threshold the first time users interact with tagged objects. Apart from the novelty factor of the technology, the first interaction is not always immediately clear or self-explaining and users have different problems with it. In addition, mobile interaction with tagged objects becomes more complex as it evolves from simple interactions with single tags, e.g. to open a website on the mobile device, to more complex interactions with multiple tags, e.g. to assemble parameters for buying a movie ticket (see Figure 1).

The increasing complexity of this new kind of mobile interaction requires better introduction and more effective assistance as its focus moves from mobile devices to physical objects and interfaces. So far, there are no standards for helping users overcome initial difficulties in the interaction with tagged objects. The goal of this paper is to increase the accessibility and usability of NFC/RFID-based mobile interaction with physical objects by improving its learnability and guidance. It investigates 4 different ways to increase the learnability of mobile interaction with tagged objects (dedicated start-tag, visual cues on physical objects, on mobile devices and on both) and 4 different ways to improve the implicit and explicit guidance in its applications. The results provide best practices for the design and implementation of more usable mobile applications that use NFC/RFID-based interaction. 


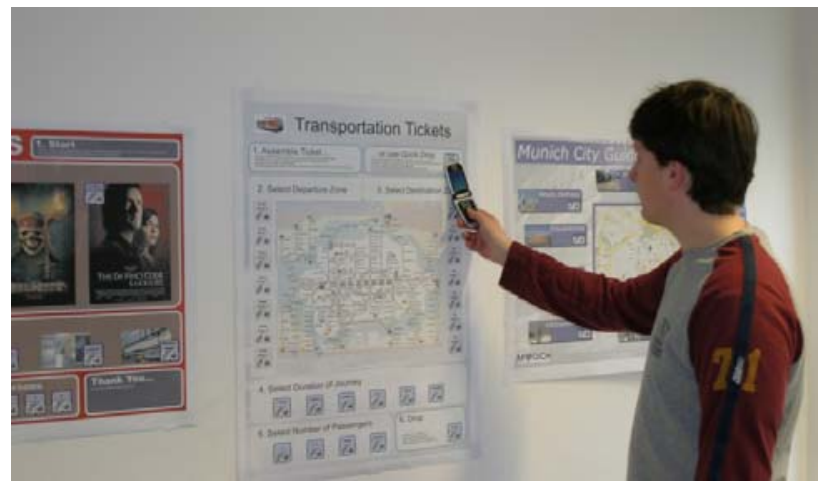

Figure 1: Mobile interaction with physical objects that comprise multiple NFC/RFID-tags

The next section gives an overview of related work on mobile interaction with NFC and RFID, focusing on the assessment of its accessibility and usability. Section 3 presents different designs for mobile and physical interfaces to improve the learnability and guidance of NFC/RFID-based interaction. Section 4 describes the setup and results of a study that has compared and evaluated these designs. Section 5 provides a summary and discussion of the major results and section 6 concludes this paper.

\section{RELATED WORK}

Research on using NFC and RFID to link the physical and the digital world and to facilitate mobile interaction has considerably evolved during the last years: The first systems have implemented contactless mobile interaction with RFID-tags and -devices, while current applications rely on NFC more often. This development is due to the increasing availability of mobile phones with built-in NFC-readers, e.g. the Nokia 6131 NFC. NFC is compatible with existing RFID infrastructures and standards, e.g. for contactless smartcards like Mifare or FeliCa [17]. It is also more flexible than RFID and aimed at mobile devices that can support different NFC interaction modes (read/write, tag emulation, P2P).

In 1999, Want et al. [16] presented some of the first examples for linking everyday objects (e.g. books, documents, business cards, watches) and corresponding digital information (e.g. electronic documents, URLs, email-addresses, electronic calendar) by using RFID-tags. Since then, many Ubicomp applications have relied on interaction with tagged objects to facilitate the interaction with associated information and services. Examples range from mobile interaction with services (e.g. [10], [1]) to new interaction paradigms like Hovering [15] or physical hyperlinks [13].

While most of these and other examples take advantage of the simplicity of interacting with single tags, several applications already implement more complex physical interactions with multiple tags, shifting the focus of interaction from mobile devices to physical interfaces even further: Collect\&Drop for example is a generic technique for Multi-Tag Interaction that is based on the typing of information from tags to increase their interoperability and to support their collection, combination and (re)use across different physical objects and applications [2]. Sanchez et al. [12] use physical interfaces to operate multimediaplayers whose control-commands have been implemented with RFID-tags. Users can operate a player by touching these tags with their mobile devices. Finally, Hardy et al. [4] use a grid of NFC- tags as an interactive surface for applications whose GUI is projected onto the tag-grid. The mobile phone is used to interact with the tags and thus manipulate the projected interface.

Next to the technical feasibility of NFC/RFID-based mobile interactions, several studies have explored their usability and accessibility: O'Neill et al. [9] have compared NFC with twodimensional barcodes and investigated the users' adoption of NFC in their daily lives. They showed that although untrained users were faster using the barcodes, trained users could significantly improve their performance with the NFC-tags. Rukzio et al. [11] have compared the interaction techniques Touching (based on NFC), Pointing (based on a laser-pointer) and Scanning (using Bluetooth) for interaction with smart-home appliances. Touching was regarded as error-resistant, secure, quick and non-ambiguous.

Mäkelä et al. [7] have conducted a field study with RFID-tags and visual markers to investigate the usability and acceptability of these technologies and how familiar people are with them. The authors showed that most users were unfamiliar with the concepts of both RFID and visual tags and often did not know how to trigger the interaction with them. They suggest that due to this lack of familiarity, the mental model about these new technologies is still very vague which may lead to usability issues. The authors also advocate the design of standardized visual cues for better recognition of NFC- and RFID-tags and to facilitate the interaction with them as they become more common.

Geven et al. [3] have conducted several complementary studies to analyze how novice users interact with NFC-equipped mobile devices and how their experiences change when using NFC more often. Similar to Mäkelä et al., they discovered that novice users often did not know how to initiate the interaction with NFC. They were also not sure about how to align the mobile device correctly to the tag, since the position of the NFC-unit was not marked on the device. The authors suggest clarifying the correct orientation of the NFC- device and providing cues on the tag and the devices so that users know how to align them correctly. They also suggest using pictograms on physical objects to explain the interaction process.

Häikiö et al. [5] have evaluated a home care service that allows elderly people to order meals for home-delivery by touching RFID-tags for different meals on a menu with their mobile devices. This touch-based interface was especially useful for elderly people who suffered from trembling hands and could therefore hardly use the small keys of mobile devices. The results of the study showed that the touch-based interface was easy to learn for the subjects and that it can decrease the cognitive load of the interaction. Nevertheless, the authors admit that the subjects would not have been able to use the system in a proper way without some assistance and training, especially about how to align mobile phones and tags correctly.

A preliminary study on the usability of NFC-based interaction investigated visual cues on tagged posters for mobile ticketing by numbering the different steps of the interaction process [2]. All subjects approved the numbering and a given order of interaction. This guidance was seen as easy, intuitive, error-preventing and helpful for novice users. Some subjects also stated explicitly that on the one hand it was useful to provide numbering but that on the other hand it was also convenient that they did not have to follow this guidance too strictly and could also select tags arbitrarily. 


\section{INTERFACE DESIGN FOR LEARNABILITY AND GUIDANCE}

Different examples and studies have shown that NFC and RFID can facilitate mobile interaction with physical objects. Nevertheless, they have also shown that the accessibility and usability of this new kind of interaction can be limited by various constraints and that users can benefit from additional assistance. This section presents different designs for mobile and physical interfaces that have been developed to lower the initial inhibition threshold of NFC/RFID-based mobile interaction with tagged objects, to make it more accessible for novice users and to facilitate the interaction with complex physical interfaces.

\subsection{A Standard Use Case Application}

In order to be able to compare different designs for improving learnability and guidance in a study, they have been implemented with a simple application for buying movie tickets comprising a tagged poster and a mobile client application. This use case is straightforward and most people can be expected to be familiar with it. It has been successfully used in previous studies (see [2]) and is flexible enough to evaluate mobile interaction with both single and multiple tags.

The poster for this use case comprises four categories of options for buying movie tickets (see Figure 2): titles of movies (4 options), timeslots (6 options), cinemas (5 options) and number of visitors (4 options). All options of a category are grouped and visually separated from the other options. For each option, there is an NFC-tag attached to the back of the poster. On its front, the position of each tag is marked with a standardized NFC-symbol (see Figure 4). Users have to touch it with their mobile devices to interact with the underlying tag and to select its option. Tags can be selected in arbitrary order. The design of the poster is as plain as possible in order not to distract users with fancy graphics. The grey box in Figure 2 is not part of this design, but indicates the position of different cues for the interaction with the poster that will be introduced in the following sections.

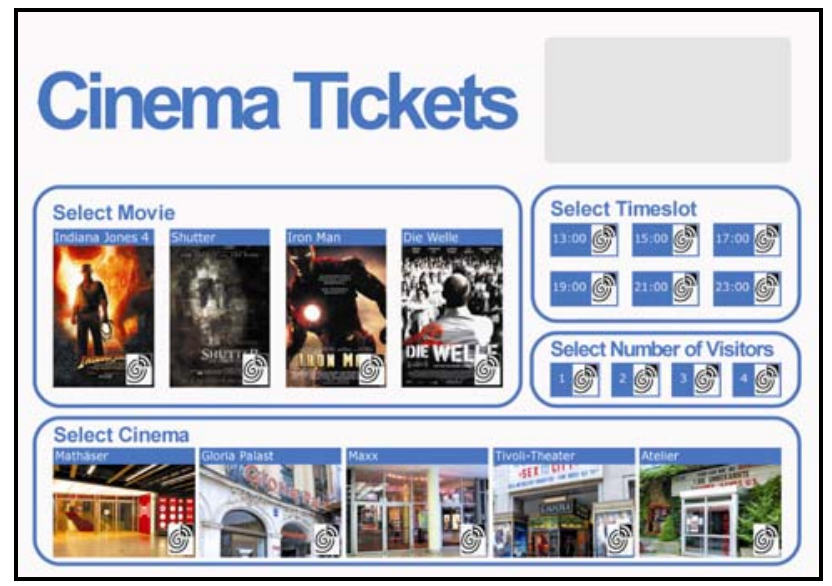

Figure 2. Standard poster design for buying cinema tickets.

In addition to the poster, a simple Java ME application has been implemented for the Nokia 6131 NFC mobile phone to display the content of selected tags. It implements the NFC push registry that automatically launches the application upon touching tags on the poster. The design of the application's interface is also deliberately plain and shows an empty list with the categories from the poster (see Figure 3a). As users interact with the poster and select different options, they are added to the respective category in the list (see Figure 3b). Users can correct false selections by touching other options from the same category on the poster. As soon as the user has selected one option for each category, a submit-command appears to finish the interaction. In the following sections, different aspects of this design will be altered to investigate their effects on the learnability and guidance of mobile interaction with tagged objects.

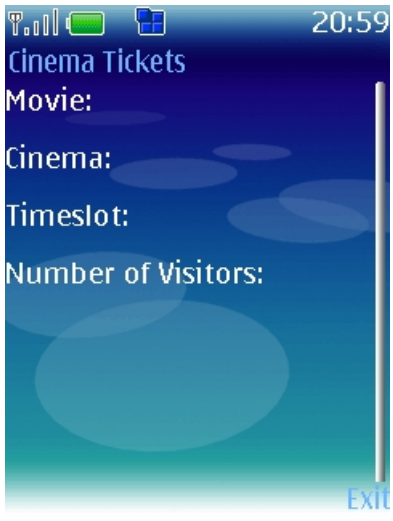

a)

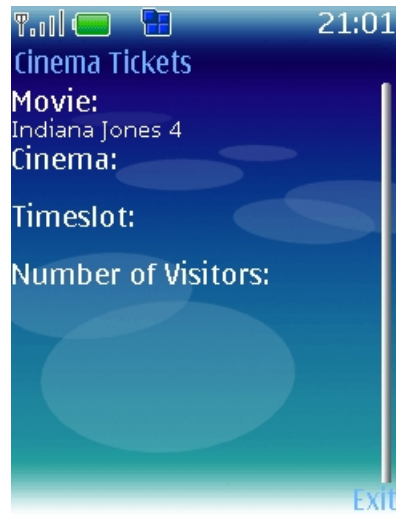

b)
Figure 3. Mobile application interface for collecting information from NFC-tags on the use case poster

\subsection{Learnability}

According to Nielsen [8], learnability is an aspect of usability that assesses the quality of a system according to how easily novice users can carry out basic tasks the first time they encounter it. Several studies have shown that novice users, who have never encountered tagged objects, often don't know how to start the interaction with them (see section 2). The following sections suggest different cues on mobile and physical interfaces to help users with this first step, thus lowering the initial inhibition threshold of NFC/RFID-based interaction. Although these cues will be evaluated with a poster for interaction with multiple tags, they can also be applied to the interaction with only one tag. Mobile interactions with single and multiple tags may vary in the total number of tags that users interact with, but both need to facilitate the step of touching the first tag in the same way.

\subsubsection{Dedicated Start-Tag}

Many applications that use NFC/RFID-based interaction mark the interactive tag on the physical object with a visually striking label (e.g. "Touch Here!”) or other cues to attract the attention of users. Therefore, the first design for increasing the accessibility of interaction with tagged objects is a dedicated start-tag that gives users an explicit starting point for further interaction.

The start-tag uses the same symbol for NFC-tags as the other tags on the poster (see Figure 4), but is four times bigger than them and fills this area with four NFC-tags. In contrast to the other learnability-designs, users have to touch one of these tags first, to open the mobile application for further interaction. Although 
users have the additional effort of touching this tag compared to the other designs, the dedicated start-tag provides a very explicit introduction to the application for the user. It is placed in the topright corner of the use-case poster (see grey outline in Figure 2) and is visually emphasized by a red border that also encapsulates a headline and a short text about how to interact with the tag (see Figure 4). Although users did not pay much attention to similar explanations in earlier studies (see [2]) the flashy coloring of the start-tag might catch their attention and direct it to the text.

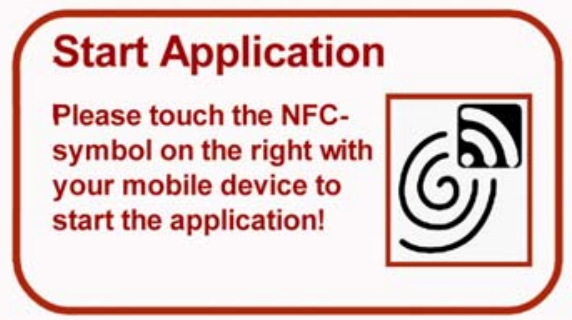

Figure 4. Dedicated start-tag with an enlarged NFC-symbol to initiate interaction with a tagged physical object

\subsubsection{Visual Cue on the Physical Object}

In contrast to the start-tag with its textual description, the next cue uses a graphic visualization that shows users exactly how to touch NFC-tags with a mobile device in order to interact with them (see Figure 5). This visual cue may be more effective with users who ignore textual cues or who simply don't know where the NFCunit is located on a device - a common source of errors in NFC/RFID-based interaction. Unlike the start-tag, the visual cue is not interactive and users can touch any of the other tags on the poster to start the interaction with the mobile application.

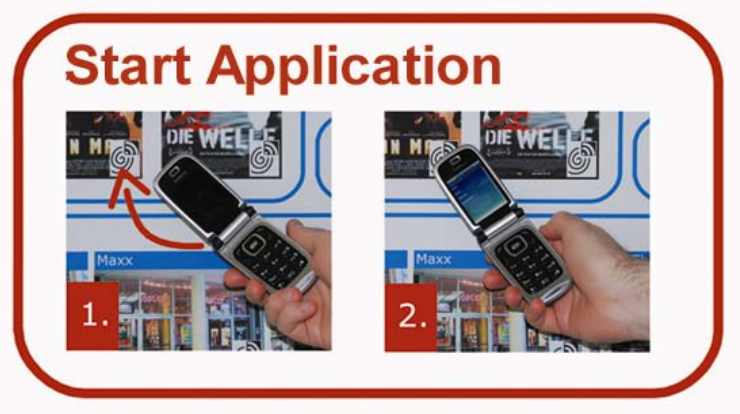

Figure 5. Visual cue that shows users how to interact with NFC-tags on the use case poster

Like the start-tag, the visual cue is placed in the top-right corner of the use-case poster and framed with a red border. In order to communicate the interaction with the poster's tags in the most unambiguous way, the visual cue comprises two photos that show the correct interaction with them. This realistic visualization was chosen because it is the closest to the actual interaction and should help users make a connection with it more easily. The first photo shows a user's hand holding a mobile phone with a dark screen next to a tag but without touching it. An arrow points from the phone to the tag indicating that the user has to touch it. The second picture shows how the user correctly touches the tag with the tip of the phone, where the NFC-unit is located. Upon touching the tag, the screen of the phone is illuminated to indicate that the phone responds to the correct execution of the interaction.

\subsubsection{Visual Cue on the Mobile Device}

Another way to show users the correct interaction between tagged objects and mobile devices is to give them cues on the latter. For example, the Nokia 6131 NFC is shipped with a transparent foil on the outer screen of its clamshell-design that shows users how to touch NFC-tags with the tip of the phone - where the NFC-unit is located - to interact with them (see Figure 6a). This cue often remains unrecognized due to its inconspicuousness and disadvantageous position.

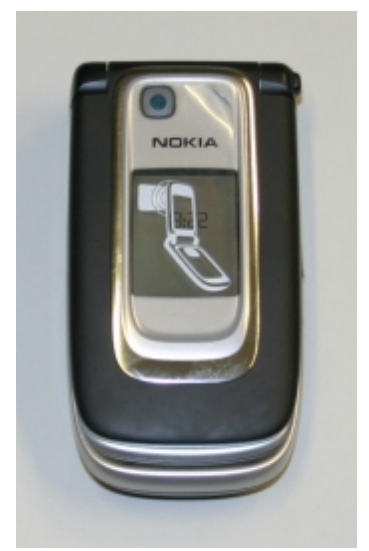

a)

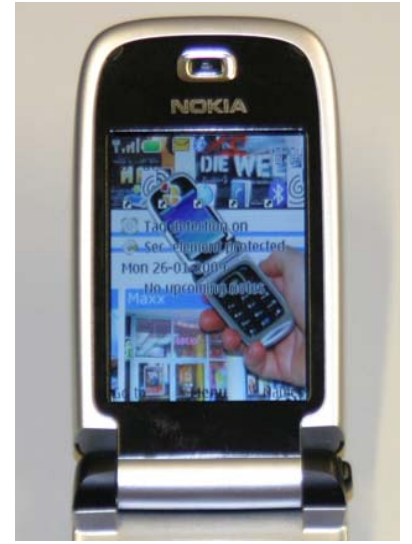

b)
Figure 6. Different cues for NFC-based interaction on mobile devices: foil on the outer screen of the Nokia 6131 NFC (a) and a visual cue as desktop background (b)

NFC/RFID-based mobile interaction is always divided between mobile devices and physical objects. In order to investigate which of them can support learnability in a better way, this design does not provide any assistance on the poster but puts a visual cue on the background of the mobile phone's main screen where users are most likely to see it (see Figure 6b). A possible design for this wallpaper-like cue may have been the NFC-symbol which also marks the position of tags on the poster. However, it was decided to use the second picture from the previous design that shows how to touch an NFC-symbol/tag correctly at one glance. That way, users can make the mental connection from this cue to the actual interaction with the poster more easily.

\subsubsection{Cues on Mobile Device and Physical Object}

The fourth design is a combination of the last two designs and uses both the visual cues on the poster and the mobile phone. This design was added in order to investigate whether there is a cumulative effect that increases the learnability of NFC/RFIDbased interaction more than the single designs.

\subsection{Guidance}

After users have learned about the basic interaction with tagged objects, applications can provide different kinds of guidance through the remaining interaction process. This is especially helpful for the novel and unfamiliar interaction with multiple tags. Efficient guidance results in short task execution times without interrupting the users' mental workflow. Since too much guidance may disrupt users, one goal is to find out how much guidance is 
necessary and which kinds of guidance stress them too much. One way to investigate guidance in NFC/RFID-based interaction is to distinguish between guidance provided by the physical object and by the mobile device. In addition, the following sections distinguish between designs for implicit and explicit guidance on each of them and thus cross-combine all four aspects for the user study. All designs use an explicit start-tag (see section 3.2.1 and Figure 4) to launch the mobile application.

\subsubsection{Guidance on the Physical Object}

The designs for improving the guidance on physical objects extend the idea of explicitly numbering the different interaction steps from [2] and compare it against a more implicit visual design of the physical object. The preliminary study has shown that numbering the steps of the interaction process was considered to be helpful for guiding users through the application. The first design adopts this idea and applies it to the use case poster. It provides explicit guidance cues by numbering the categories of its options (see Figure 7a) to give users clear hints about the order of interaction steps that they should follow.

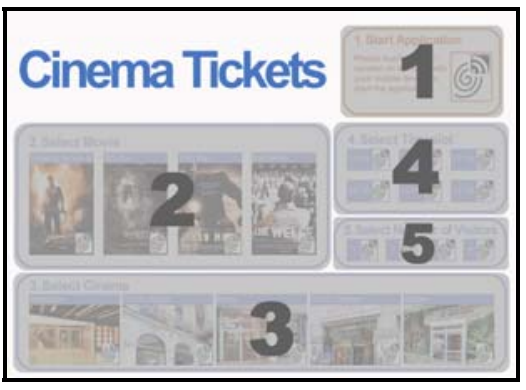

a)

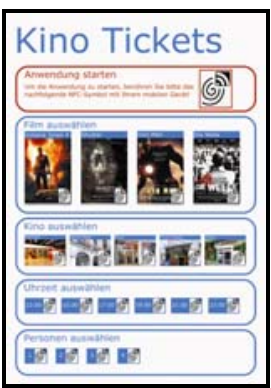

b)
Figure 7. Explicit and implicit guidance cues on the use case poster through numbering of interaction steps (a; grey boxes for better visualization) and their spatial arrangement (b)

In contrast to the explicit numbering of interaction steps, their spatial arrangement on the physical object can provide a more implicit guidance. Regarding the use case poster, arranging all categories in a vertical order from top to bottom can guide the user in an implicit way (see Figure 7b). Another option would have been to arrange the categories horizontally, so that users can follow them from left to right. However, the vertical arrangement of interaction steps was preferred as it seemed to be less ambiguous and more straightforward.

\subsubsection{Guidance on the Mobile Device}

Mobile applications have better possibilities to actively influence users and guide them through an interaction process. Hence, the first design for guidance on mobile devices is an explicit step-bystep instruction that tells users what to do, similar to a wizard in desktop applications. After the user has started the mobile application by touching the start-tag on the poster, it does not display the entire list of categories. The wizard shows only the name of the next category for which the user should select a tag and asks him to do so explicitly (see Figure 8a). If the user selects a tag from another category, the wizard shows a warning, asking him to select an option for the current category (see Figure 8b). This strict procedure may limit the flexibility of the application to select tags in arbitrary order and seem to be overwhelming for some applications. Nevertheless, this design makes sure that users accomplish the interaction correctly without missing a step.

In order to find out whether users appreciate the explicit guidance provided by the wizard-design, the study will compare it against the list of categories from the mobile application (see section 3.1 and Figure 3). This interface is very simple and only assists the user by displaying the categories for which he has to select options, respectively touch tags on the physical objects, which can be seen as an implicit way of guidance. For the user study, both designs will be tested with each design for guidance on the physical object, respectively the use case poster.

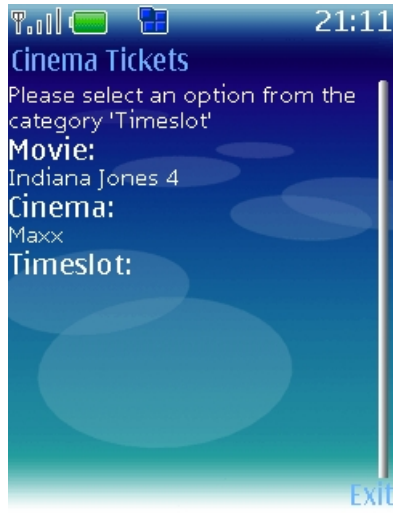

a)

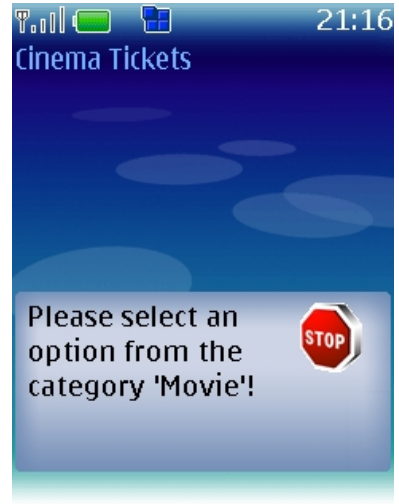

b)
Figure 8. Explicit guidance on mobile devices with a wizard

\section{USER STUDY AND EVALUATION}

In order to evaluate the impact of the presented designs on the learnability and guidance of mobile interaction with tagged posters, a user study was conducted to compare them. The next sections present the setup of the study as well as its results.

\subsection{Experimental Design}

The comparison and evaluation of the different designs for learnability and guidance used an independent measures study design that tested subjects in independent groups. In order to estimate the possibilities of these designs to effectively lower the initial inhibition threshold of NFC/RFID-based interaction in a realistic way, the study was conducted with 40 subjects who had no previous experience with this kind of interaction.

The average age of all subjects was 23.7 (from 18 to 38); 25 were male, 15 female. Among the subjects were 18 students of (media) computer sciences, 17 students of non-technical subjects, 4 pupils and 1 educator. They have owned a mobile phone for an average of 7 years. 18 subjects have heard of NFC or RFID before, but no subject has used them before. All subjects were randomly assigned to different groups to test one of four designs for learnability and one of four designs for guidance to avoid learning effects. Subjects with a technical background were equally distributed between them. All tests were conducted with prototypes based on the standard design for tagged posters and mobile applications presented in section 3.1.

The study comprised two parts: The first part compared the different learnability-designs that were presented in section 3.2.: 
poster with dedicated start-tag, poster with visual cue, mobile device with visual cue and visual cues on both mobile device and poster. In order to evaluate their impact on novice users, all subjects were randomly assigned to one of 5 groups to test each of the designs independently. The fifth group served as the control group that did not receive any help at all. For the second part of the study, all subjects from each learnability-group were randomly assigned to 4 groups to test the cross-combinations of implicit and explicit guidance on mobile devices (list and wizard) and physical interfaces (vertical arrangement and numbering) that were presented in section 3.3.

\subsection{Hypotheses}

Before the study, the following hypotheses were made about the impact of the presented designs on the learnability and guidance of mobile interaction with tagged objects:

- Hypothesis H1: All learnability designs help users to understand interaction with tagged objects better and improve its learnability compared to providing no help at all.

- Hypothesis H2: A dedicated start-tag on the physical object improves learnability more than designs without one.

- Hypothesis H3: A visual cue on the physical object improves learnability more than one on the mobile device.

- Hypothesis H4: Visual cues on both the physical object and the mobile device improve learnability more than visual cues on either one of them.

- Hypothesis H5: Implicit guidance through the spatial arrangement of interaction steps on the physical object improves task performance compared to explicit guidance by numbering them.

- Hypothesis H6: Explicit step-by-step guidance provided by the mobile application decreases the task performance compared to implicit guidance on the mobile device.

- Hypothesis H7: Explicit step-by-step guidance provided by the mobile application increases the number of attention shifts compared to implicit guidance on the mobile device.

\subsection{Procedure}

At the beginning of the study, the investigator gave each subject a short, general introduction and asked him to fill out a background questionnaire. In order to test the impact of the different designs on the learnability of NFC/RFID-based mobile interaction in a valid way, only subjects with no previous experience in this area were used for the study. Consequently, the investigator did not give them any introduction to NFC, RFID or the Nokia 6131 NFC mobile phone that was used for the study.

All subjects started the study by testing one of the 5 learnabilitydesigns which were randomly assigned to them. The task for all 5 groups was the same: the subjects had to buy cinema tickets (for a certain movie/cinema/timeslot/number of visitors) using the tagged poster and the mobile application. In each group, the poster was attached to the backside of a pin board, which was flipped over after the investigator had given the subject the task description and the mobile phone (see Figure 9). That way, subjects could not learn about the design of learnability cues before the time measurement started. After a subject had completed the learnability-task, he had to fill out a questionnaire about the tested design. During that time, the investigator could exchange posters for the following guidance-test.

The second part of the study tested the different designs for guidance in a similar way: The subjects were again asked to buy cinema tickets using the tagged poster and the mobile application. All poster designs for testing guidance comprised the dedicated start-tag. In order to assure consistency for all subjects in the guidance-test, the investigator showed them on a separate poster how the start-tag works. Afterwards, the investigator again flipped the pin board with the poster and the subjects could carry out the task. All subjects finished the study by filling out a final questionnaire about the tested guidance-design.
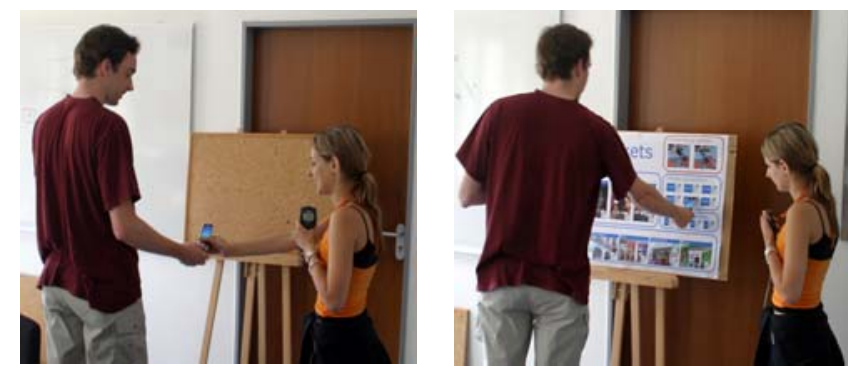

Figure 9. Setup of the user study: the investigator controls the presentation of designs for learnability and guidance to avoid preliminary learning effects

\subsection{Measurements}

The dependent variables for evaluating the performance of the subjects and thus the impact of the different designs on learnability and guidance were task execution time, number of errors and number of attention shifts (i.e. a shift of attention from the mobile device to the physical interface or back, see [5]). During the study, all subjects were videotaped and the mobile application recorded the number of touched tags. Errors and attention shifts were measured by a careful post-hoc analysis of the recorded material.

The task execution time was measured in two steps: The first step measured the time from the beginning of the interaction, when the poster was flipped over and the subject learned about its design, until the first tag on the poster was touched. The second step measured the time until finishing the task. The time for this step was recorded by the mobile application, while the time before touching the first tag was measured with a stop watch. In addition, the application also recorded the time between touching the first and the second tag for analyzing the time between touching the dedicated start-tag and touching tags for regular options.

\subsection{Results}

\subsubsection{Learnability - Performance}

An improvement in learnability was defined as an enhancement in task performance that can be measured by faster execution times and less errors made by the subjects. Figure 10 shows the mean times and standard deviations for touching the first and second tag on the poster as well as the total task execution time for all 5 tested learnability designs, including the control group.

The time it took the subjects to touch the first NFC-tag (blue bars in Figure 10) is most interesting for estimating the impact of these 
designs on the learnability of NFC/RFID-based interaction: The control group clearly performed worst (mean=02:38; standard deviation=01:30; all times in min:sec), while the dedicated starttag performed better than any other design $(m=00: 51$; $s d=00: 46)$. The performance of the visual cue on the poster was slightly worse $(m=01: 14 ; s d=00: 48)$, followed by the visual cue on the mobile phone ( $m=01: 28$; $s d=00: 54)$. It can be assumed, that the visual cue on the poster attracts the subjects' attention more than the small image on the mobile phone. This result also indicates that the attention of the subjects was more focused on the physical interface than on the mobile interface. Surprisingly, the combination of both visual cues performed worse $(\mathrm{m}=01: 53$; $\mathrm{sd}=00: 50$ ) than the designs with cues on either the poster or the mobile device. Some subjects seemed to be confused by both cues as they tried to find a connection between them, e.g. by touching the visual cue on the poster with the mobile phone's display.

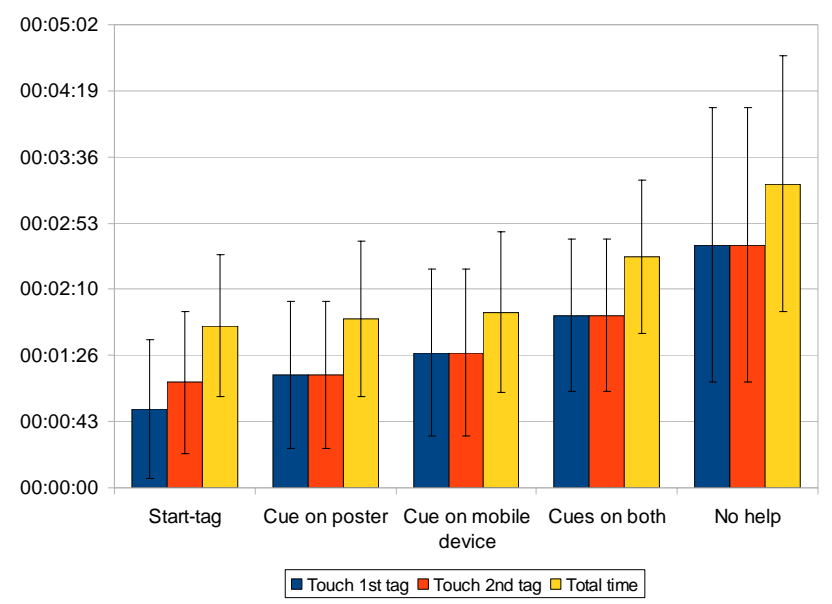

Figure 10. Task execution times for all learnability designs

A one-way independent ANOVA was used to analyze the performance of all designs regarding the interaction with the first tag. When all designs were compared against each other, using the Scheffe test, a significant difference was only found between the dedicated start-tag and the control group.

The total task execution time (yellow bars in Figure 10) showed the same order of performance for the different designs: dedicated start-tag ( $m=01: 46$; $s d=00: 46)$, visual cue on the poster $(m=01: 50$; $s d=00: 51)$, visual cue on the mobile device $(m=01: 55$; $s d=00: 52)$, on both $(\mathrm{m}=02: 31 ; \mathrm{sd}=00: 50)$ and no cues at all $(\mathrm{m}=03: 18$; $\mathrm{sd}=01: 23$ ). After the subjects had overcome the initial inhibition threshold of touching the first tag, the remaining time until the end of the interaction did not vary too much across all groups between 35 and 40 seconds - except for the visual cue on the mobile device with 26 seconds on average. These results indicate that subjects can learn this new kind of interaction very quickly and that subsequent interactions become much faster. Again, the ANOVA test showed significant differences between the mean values. When running the Scheffe test, there was again a significant difference between the start-tag and the control group.

\subsubsection{Learnability - Errors}

Subsequently, the error rates of all groups were compared against each other. An error was defined as any mistake made by the subjects, for instance when they wanted to take a picture of the
NFC-symbol with the phone-cam, or any hint that had to be given by the investigator during the execution of the task. The mean numbers of errors for all designs (see Figure 11) emphasize the previous results: The dedicated start-tag $(\mathrm{m}=2.37$; $\mathrm{sd}=2.32)$ performed better than the visual cues on the poster $(m=3.62$; $\mathrm{sd}=2.19)$, on the mobile phone $(\mathrm{m}=4.37$; $\mathrm{sd}=3.02)$ and on both $(m=6.25$; $s d=2.43)$, while the control group made the most errors on average $(m=7.25 ; \mathrm{sd}=3.19)$. The one-way independent ANOVA showed highly significant differences in error rates. However, the post-hoc Scheffe test discovered again a significant difference only between the start-tag and the control group.

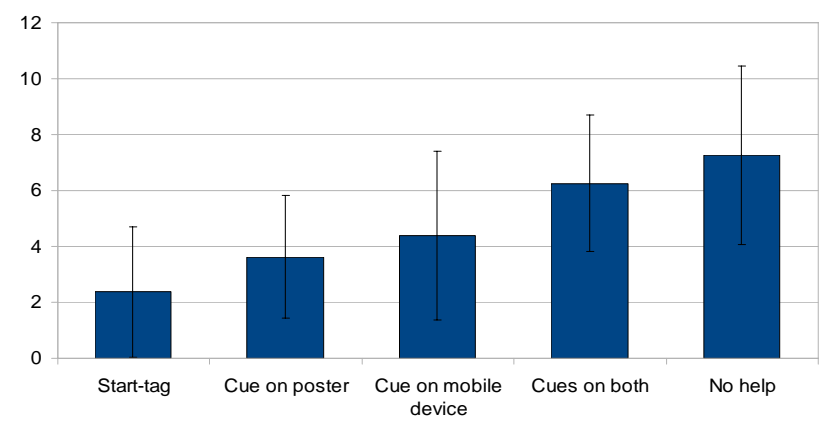

Figure 11. Mean number of errors for all learnability designs

Apart from the average number of errors, it is interesting to look at the different kinds of errors that were made: $60 \%$ of all subjects thought that they needed to use the mobile phone's menu, either to activate NFC (which was switched on by default), to connect to the Internet, or to launch the application. The blue bars in Figure 12 represent all subjects who made an error by opening the menu. Again, subjects who have used the dedicated start-tag performed best, followed by visual cues on the poster and the mobile phone. All subjects in the last two groups opened the application menu before they realized how the interaction really worked. This confirms the assumption that the start-tag gives subjects the best idea of how to launch the application.

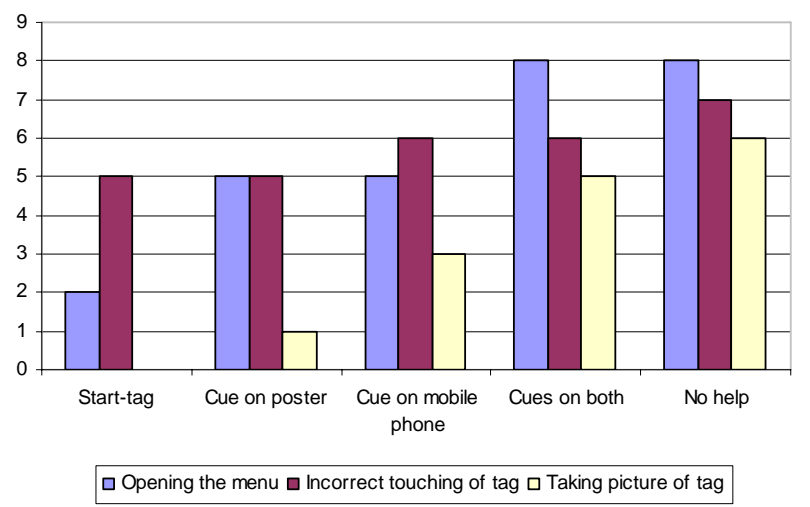

Figure 12. Most common errors during mobile interaction with tagged poster for all learnability designs

Sooner or later all subjects realized that they had to touch the NFC-symbols with their mobile phones to interact with them. However, $73 \%$ of them made a mistake in the way they touched the NFC-symbols (see violet bars in Figure 12). The order of 
performance for the different designs remains the same, although the differences are not as large as before. Errors include touching tags with the wrong part of the mobile phone, especially its bottom instead of the tip, holding the mobile phone too far away from tags to read them, touching tags too shortly to read them, trying to touch the NFC-symbols with the finger instead of the phone or touching completely wrong parts on the poster, like the visual cue or the movie posters instead of their corresponding NFC-symbols. Many subjects were also insecure about how to interact with the poster and did not think that touching a paper poster with their mobile devices would trigger any action.

Surprisingly, $38 \%$ of all subjects tried to take a picture of the NFC-symbol with the mobile phone's camera to interact with it, as they confused it with some kind of 2D-barcode. Some subjects only mentioned this idea and asked if they should take a picture, simply as this seemed to be the only way left to interact with the poster. As Figure 12 shows, no subject from the first group and only one from the second group wanted to take a picture of the NFC-symbol, while this was the case for more than half of the subjects in the fourth and the fifth group.

\subsubsection{Learnability - User Feedback}

The study also collected qualitative data from questionnaires, user feedback and general observations made by the investigator. The questionnaires used Likert-scales to evaluate the level of agreement with different statements, ranging from 1 ("totally disagree") to 5 ("totally agree").

The first question asked the subjects whether launching the mobile application was immediately clear to them. The agreement with this statement was quite low. Nevertheless, this seemed most obvious to subjects who had used the start-tag $(\mathrm{m}=2.6$; $\mathrm{sd}=1.5)$, followed by the visual cue on the poster $(m=1.9$; $s d=0.8)$, visual cues on the poster and the mobile device $(\mathrm{m}=1.9$; $\mathrm{sd}=0.8)$, the control group $(\mathrm{m}=1.6 ; \mathrm{sd}=1.1)$ and the visual cue on the mobile device $(\mathrm{m}=1.4$; $\mathrm{sd}=1.1)$. Some subjects did not believe that the poster was actually interactive, wanted to launch the application from the mobile phone's menu, did not understand the pictures of the visual cues or wanted to take a picture of them.

The next question asked whether launching the application from the application's menu would have been easier for them. Subjects who had used the start-tag agreed the least $(\mathrm{m}=2.0$; $\mathrm{sd}=0.9)$, followed by visual cues on the mobile device $(m=2.9$; $\mathrm{sd}=1.6)$, on the poster $(m=3.0$; $s d=1.7)$, on both of them $(m=3.5$; $s d=1.3)$ and the control group $(\mathrm{m}=4.0$; $\mathrm{sd}=0.8)$.

Another question asked whether there were enough hints that explained the interaction with the tagged poster. Once more, the first group had the fewest problems $(\mathrm{m}=4.3$; $\mathrm{sd}=0.5)$, followed by visual cues on the poster $(\mathrm{m}=3.1$; $\mathrm{sd}=1.1)$, on poster and mobile device $(\mathrm{m}=2.4$; $\mathrm{sd}=1.1)$, on the mobile device $(\mathrm{m}=2.3$; $\mathrm{sd}=1.3$ ) and the control group $(\mathrm{m}=1.8 ; \mathrm{sd}=1.4)$. This indicates that the textual cues of the start-tag provide better help for the interaction with tagged objects than all designs that used visual cues.

\subsubsection{Guidance - Performance}

The comparison of guidance-designs tried to find the best way to guide the subjects through the interaction with the tagged posters without interfering too much with their mental workflow. Hence an improvement in guidance would result in better task execution times and less attention shifts. Therefore, the total time it took the subjects to perform the specified task is most interesting. Figure 13 shows that there were no noticeable differences in the performance for touching the start-tag and the first option-tag (blue and orange bars) across all designs. Similarly, ANOVA tests showed no significant differences regarding these two measurements. Their values are much lower than in the learnability-group that tested the start-tag - also because the investigator told all subjects how it works, so that subjects who already knew it from the learnability-test had no advantage.

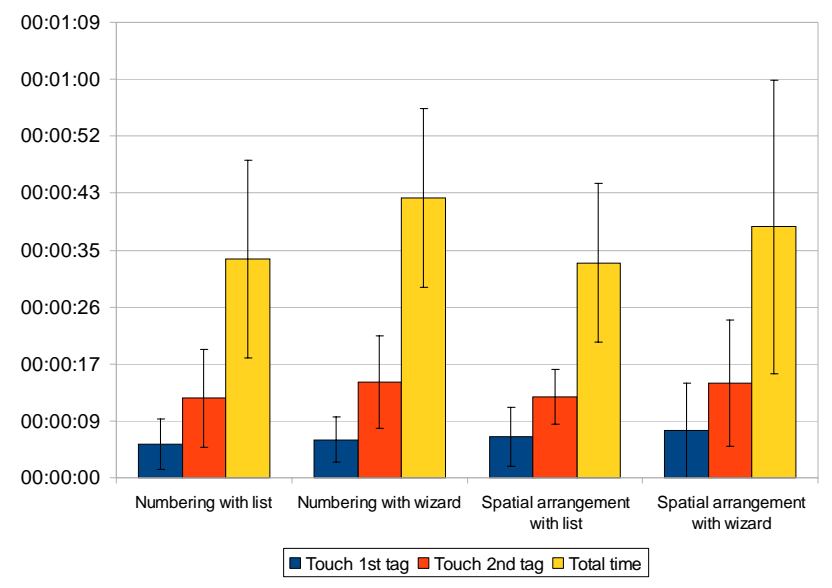

Figure 13. Task execution times for all guidance designs

The total task execution times of all guidance-designs (yellow bars in Figure 13) are much better than for the same task in the learnability-designs. The combination of implicit designs (spatial arrangement of options and mobile list interface) performed best, while the combination of explicit designs (numbering of options and mobile wizard interface) performed worst. Figure 13 also shows better task execution times for the designs with implicit guidance on the mobile device $(m=00: 33$ and $s d=00: 15$ with numbering and $\mathrm{m}=00: 32$ and $\mathrm{sd}=00: 12$ with spatial arrangement) compared to the designs with explicit guidance through the wizard $(m=00: 42$ and $s d=00: 13$ with numbering and $m=00: 38$ and sd=00:22 with spatial arrangement). This indicates that the explicit guidance of the wizard puts more effort on the subjects and disrupts their mental workflow. Some subjects did not notice the wizard and made mistakes, triggering error messages which increased the total task execution time. Subjects, who have read the wizard's instructions, also spent more time looking at the phone's display. On the other hand, in the designs with the implicit list-interface, one glance at the display was enough to give feedback.

The comparison of the two designs for guidance on the poster implicit, spatial arrangement of options and explicit numbering does not yield strong results. When combined with the mobile list-interface, both poster-designs performed almost equally well. When combined with the mobile wizard interface, the spatial arrangement of options performed slightly better than their numbering. A one-way independent ANOVA of execution times between all designs also showed no significant differences.

The study also measured attention shifts to find effects on the task execution time. A (macro-) attention shift is defined as a context switch between the mobile phone's display and the poster [5] 
Subjects who tested the mobile wizard interfaces could not arbitrarily interact with the tagged poster but had to follow the wizard. Thus they can be expected to look at the mobile device more often, resulting in more attention shifts. This is confirmed by the results of the analysis: On the one hand, the interaction with the numbered options resulted in the lowest number of attention shifts when used without the wizard $(m=6.2 ; \mathrm{sd}=3.2)$ and the highest number when used with the wizard $(m=9.6$; $\mathrm{sd}=1.3$ ). On the other hand, the same comparison based on the spatial arrangement of options does not confirm these results as both numbers of attention shifts are basically identical $(m=7.6$; $\mathrm{sd}=4.1 / 3.5$ ). These results indicate but do not directly confirm that the explicit guidance of the mobile wizard interface automatically increases the number of attention shifts and thus the duration of the task performance. Since almost all subjects followed the guidance on the poster, they did not necessarily have to read the wizard's instructions and hence switch attention. Regarding the one-way independent ANOVA, no significant differences could be found concerning the users' attention shifts.

\subsubsection{Guidance - User Feedback}

The study also collected user feedback about the guidance designs using questionnaires with Likert-scales from 1 ("totally disagree”) to 5 ("totally agree"). One question asked the subjects whether they always knew what step to take next during the execution of the task. Subjects who tested the implicit arrangement of options on the posters agreed more to this question than subjects who tested the explicit numbering of options. Similarly, the subjects gave the implicit list interface on the mobile device better ratings than the explicit wizard. Again, the combination of implicit designs (visual arrangement of options and list) performed best $(m=4.9$; $s d=0.3)$, while the combination of explicit designs (numbering of options and wizard) performed worst $(\mathrm{m}=3.8$; $\mathrm{sd}=0.9$ ). Although the wizard should help subjects to know what step to take next, it seems to have confused some of them and to have provided too much guidance. Some subjects complained about having to stick to the wizard's predefined order of interaction and often did not notice its error messages.

The subjects were also asked whether they preferred to be guided by the mobile device or by the poster. $77 \%$ of the subjects across all groups preferred guidance by the latter, which was seen as faster than guidance on the mobile device and provided the bigger and more lucid interface for the application. $17.5 \%$ of the subjects preferred guidance on the mobile device out of habit and because they did not want to interact with a poster in public.

Finally, the study investigated whether the subjects stuck to the order of interaction steps, that was indicated by the arrangement of options on the posters (see Figure 7) as well as the mobile list interface (see Figure 3). All subjects that tested the implicit order of options on the poster from top to bottom stuck to this order while most of the other subjects did not follow the explicit numbering of options. The mobile interface did not have a noticeable impact on these results. This indicates that the subjects often did not pay attention to the wizard and hence did not follow the predefined order more often than the other subjects. In fact, the subjects seemed to follow the guidance on the physical object much more than on the mobile device. The analysis of the video showed that the subjects that did not follow the given order chose various own ways of selecting the different options.

\section{SUMMARY AND DISCUSSION}

The study showed that all designs for improving the learnability of mobile interaction with tagged objects performed better regarding time performance and number of errors than the control group that did not get any help. This confirms hypothesis H1, although there are only significant differences between the dedicated start-tag and the control group. Similarly, the start-tag performed best among all learnability-designs, regarding both time performance and error rate which confirms hypothesis H2.

Although the differences were not significant, the visual cue on the poster always performed better than the visual cue on the mobile phone. Although hypothesis $\mathrm{H} 3$ can not be definitely proven by these findings, they strongly indicate that a visual cue on the physical object is more helpful for the user than on the mobile phone's small display. This result already anticipates the more general observation that the subjects preferred interacting with the physical object, which was confirmed by their feedback.

Hypothesis H4 has to be fully rejected. The combination of visual cues on both the poster and the mobile device did not have a cumulative effect, but even performed worse than both designs using only one of these cues each. In the study, the subjects seemed to be confused by both visual cues and their redundancy. Regarding the question whether textual or visual cues are more helpful, all visual cues always performed worse than the start-tag with its textual cues although this is difficult to generalize.

These results are also supported by the analysis of errors and user feedback. Again, the dedicated start-tag lowers the initial inhibition threshold of NFC/RFID-based mobile interaction the most for novice users, followed by cues on the poster, the mobile device and on both, while the control group performed worst in most cases. Although the different designs have noticeable effects on the learnability of NFC/RFID-based interaction, the number of errors is still quite high, especially during the initial phase of the interaction. Among various errors, $60 \%$ of all subjects wanted to start the interaction by opening the application's menu, 73\% of them made mistakes in the way they touched the NFC-tags and surprisingly $38 \%$ of them confused the NFC-symbol with a visual marker and wanted to take a picture of it.

The study supports hypothesis $\mathrm{H} 5$ as the implicit vertical arrangement of interaction steps on the physical object showed slightly better performances in task execution times than their explicit numbering. The comparison of mobile interfaces regarding their impact on guidance provides more noticeable results than the comparison of physical interface designs: subjects who used the explicit step-by-step guidance of the mobile wizard application performed worse than subjects who used the implicit mobile list interface, confirming hypothesis H6. The wizard application turned out to be more distracting for the subjects than it was helpful. This result is partially confirmed by the number of attention shifts for all guidance-designs. However, hypothesis H7 can only be partially confirmed, as the usage of the mobile wizard interface only showed an increase in the number of attention shifts when combined with explicit guidance on the physical objects, but not with the implicit one.

Again, these quantitative results are supported and confirmed by qualitative user feedback. In summary, the combination of implicit designs (spatial arrangement of interaction steps on the physical objects and mobile list interface) performed better than 
the combination of explicit designs (numbering of interaction steps and mobile wizard interface) regarding task execution time and user feedback. In addition, the explicit step-by-step guidance provided by the mobile wizard interface always performed worse than the implicit list interface, providing too much guidance, putting more effort on the subjects and disrupting their own mental workflow. Compared to previous evaluations [2] this study cannot confirm any preferences for the numbering of interaction steps on physical objects. In general, subjects preferred arbitrary interaction with tags on the physical interface to controlled guidance provided by the mobile device. Applications should also implement guidance as a kind of assistance that users are not obliged to follow.

\section{CONCLUSION}

This paper has compared different designs and techniques to improve the accessibility and usability of mobile interaction with tagged objects through learnability and guidance. The results of their evaluation show that these designs can effectively lower the initial inhibition threshold of NFC/RFID-based mobile interaction and have an impact on their learnability and guidance. The design of the dedicated start-tag that provides an explicit starting point for this interaction clearly performed best, compared to different visual cues on physical objects and the mobile device. In addition to quantitative and qualitative measurements, the results also showed the most common mistakes that occurred in mobile interaction with tagged objects. These and other results may be used as best practices to guide the future development of physical mobile applications.

The paper tried to cover an appropriate range of designs for improving the learnability and guidance in mobile interaction with tagged objects. Nevertheless, this selection is inherently limited and many factors that affect this interaction remain to be explored. The focus of mobile interaction shifts to physical objects whose design may influence this interaction in many more ways than were presented in this paper, e.g. regarding the visual design or layout of interactive elements on physical interfaces.

While the study was conducted with a prototype for Multi-Tag Interaction, we hope that its results, especially regarding learnability, are also valid for mobile interaction with single tags whose applications are much more common in everyday life. Mobile interaction with tagged objects does also not only apply to interaction with mobile posters, although they are one of the major use-cases. Future work in these areas has to find out to which degree their results can be transferred to other applications.

\section{REFERENCES}

[1] Broll, G., Siorpaes, S., Rukzio, E., Paolucci, M., Hamard, J., Wagner, M., and Schmidt, A. 2007. Supporting Mobile Service Usage through Physical Mobile Interaction. In Proc. of PERCOM'07. IEEE Computer Society, Washington, DC, 262-271.

[2] Broll, G., Haarländer, M., Paolucci, M., Wagner, M., Rukzio, E., and Schmidt, A. 2008. Collect\&Drop: A Technique for Multi-Tag Interaction with Real World Objects and Information. In Proc. of AmI'08. Lecture Notes In Computer Science, vol. 5355. Springer-Verlag, Berlin, Heidelberg, 175-191.
[3] Geven, A., Strassl, P., Ferro, B., Tscheligi, M., and Schwab, H. 2007. Experiencing real-world interaction: results from a NFC user experience field trial. In Proc. of MobileHCI '07, vol. 309. ACM, New York, NY, 234-237.

[4] Hardy, R. and Rukzio, E. 2008. Touch \& interact: touchbased interaction of mobile phones with displays. In Proc. of MobileHCI '08. ACM, New York, NY, 245-254.

[5] Häikiö, J., Wallin, A., Isomursu, M., Ailisto, H., Matinmikko, T., and Huomo, T. 2007. Touch-based user interface for elderly users. In Proc. of MobileHCI '07, vol. 309. ACM, New York, NY, 289-296.

[6] Holleis, P., Otto, F., Hussmann, H., and Schmidt, A. 2007. Keystroke-level model for advanced mobile phone interaction. In Proc. of CHI '07. ACM, New York, NY, 1505-1514.

[7] Mäkelä, K., Belt, S., Greenblatt, D., and Häkkilä, J. 2007. Mobile interaction with visual and RFID tags: a field study on user perceptions. In Proc. of CHI '07. ACM, New York, NY, 991-994.

[8] Nielsen, J. 2008. Usability 101: Introduction to Usability. www.useit.com/alertbox/20030825.html.

[9] O'Neill, E., Thompson, P., Garzonis, S., and Warr, A. 2007. Reach out and touch: Using nfc and 2d barcodes for service discovery and interaction with mobile devices. In Proc. of Pervasive'07, volume 4480 of Lecture Notes in Computer Science, 19-36. Springer, 2007.

[10] Riekki, J., Salminen, T., and Alakarppa, I. 2006. Requesting Pervasive Services by Touching RFID Tags. IEEE Pervasive Computing 5, 1 (Jan. 2006), 40.

[11] Rukzio, E., Leichtenstern, K., Callaghan, V., Schmidt, A., Holleis, P., and Chin, J. 2006. An Experimental Comparison of Physical Mobile Interaction Techniques: Touching, Pointing and Scanning. In Proc. of Ubicomp 2006, California, USA. 17-21 September 2006.

[12] Sánchez, I., Riekki, J., and Pyykknen, M. 2008. Touch \& control: Interacting with services by touching RFID tags. In Proc. of IWRT 08, June 12-13 2008.

[13] Schwieren, J. and Vossen, G. 2007. Implementing Physical Hyperlinks for Mobile Applications Using RFID Tags. In Proc. of IDEAS`07. IEEE Computer Society, Washington, DC, 154-162.

[14] SmartTouch website. www.smarttouch.org

[15] Välkkynen, P. 2006. Hovering: Visualising RFID Hyperlinks in a Mobile Phone. MIRW 2006.

[16] Want, R., Fishkin, K. P., Gujar, A., and Harrison, B. L. 1999. Bridging physical and virtual worlds with electronic tags. In Proc. of CHI '99. ACM, New York, NY, 370-377.

[17] Want, R. 2006. An Introduction to RFID Technology. IEEE Pervasive Computing 5, 1 (Jan. 2006), 25.

[18] Weiser, M. 1995. The computer for the 21st century. In Human-Computer interaction: Toward the Year 2000, R. M. Baecker, J. Grudin, W. A. Buxton, and S. Greenberg, Eds. Morgan Kaufmann Publishers, San Francisco, CA, 933-940. 